\title{
THE EFFECT OF CHRONIC ADMINISTRATION OF AMPICILLIN/ SULBACTUM ON RAT DEVELOPING ENAMEL
}

\author{
Amr Koriem Fatouh*, Dahlia Ghazy Mohamed Rateb ${ }^{* *}$ \\ and Nuha Abdul Fattah Abdullah Baraka
}

\begin{abstract}
Objective: This study aimed to evaluate the effect of chronic administration of Ampicillin/ Sulbactum on enamel development of Albino rats' mandibular first molar.

Materials \& Methods: Eight pregnant female Albino rats were equally divided into two groups: control and treated group. The treated group received intraperitoneal injection of 200 $\mathrm{mg} / \mathrm{kg}$ BW of Ampicillin/Sulbactum once daily for nine days till delivery. This was followed by injection of the offspring for additional 11 days. Treated offsprings $(n=7)$ were sacrificed at 12 days of birth and H\&E stained sections of the mandibular first molar tooth germ were examined. The other half of the treated pups was sacrificed at 28 days of birth, their mandibular first molars were examined by quantitative computerized tomography (QCT), scanning electron microscope (SEM) $\&$ ground section statistical analysis was performed for enamel measurements of H\&E, ground sections and QCT.
\end{abstract}

Results: Examination of H\&E stained sections of the Ampicillin/Sulbactam treated subgroup at 12 days revealed an apparent reduction in enamel matrix thickness which was statistically insignificant compared with control subgroup. QCT showed a significant decrease in enamel density of the treated subgroup compared with untreated subgroup at 28 days. SEM observation showed enamel surface irregularities, pores and fissures. An insignificant reduction in enamel thickness was revealed by statistical analysis of the ground sections under light microscope.

Conclusion: Within the limitations of this study, chronic administration of Ampicillin/ Sulbactam adversely affected the development of enamel in rat's offspring.

KEYWORDS: Ampicillin/Sulbactum, Enamel, Scanning electron microscopy, Quantitative computerized tomography.

* Master Degree, Faculty of Ain Shams

** Associate Professor, Assistant Professor of Oral Biology Faculty of Dentistry, Ain Shams University *** Lecturer of Oral Biology Faculty of Dentistry, Ain Shams University 


\section{INTRODUCTION}

Tooth development is known to be regulated by a sequence of inductive and reciprocal interactions between epithelial and mesenchymal cells. The development proceeded through different stages and can be divided into morphogenesis, dental cell differentiation, secretion and mineralization of tooth specific matrices (Thesleff et al., 2001).

Tooth development is genetically controlled but is susceptible to environmental disturbances, basically, systemic factors that disturb the function of ameloblasts during their developmental stages. For the most part, the human permanent incisors and first molars mineralize during the first years of childhood and therefore they are at greatest risk for developmental enamel defects such as fluorosis and molar incisor hypomineralization (Elina Nystrom et al., 2007).

Most of enamel defects are clinically seen as white or yellow demarcated opacities that vary in size and severity, sometimes leading to enamel breakdown after eruption. Many studies have shown associations between molar incisor hypomineralization and medical problems at birth or during the first years of life. Pre-term birth and common childhood illnesses such as acute otitis media and respiratory infections were found to be associated with these defect directly or indirectly (Allazzam et al., 2014) .

The use of medication as antibiotics has been proposed as a putative aetiological factor for enamel defects. The appearance of hypomineralized enamel defects caused by antibiotics depends on the dosage $\&$ duration of antibiotic administration (Rao et al., 2016).

It was reported that appearance of extensive enamel hypoplasia could be due to prolonged antibiotics usage (Simratvir, 2011). Ampicillin/ sulbactum is one of the most prescribed broad spectrum Beta lactam antibiotics families as it is considered to be safe in pregnancy and childhood period since physicians proved that this family of antibiotics had little or no effect on mother or children (Adam, 2002). The usage of Ampicillin/ Sulbactam is indicated in pneumonia with high suspicion of anaerobic component, also rheumatic fever where it is used chronically. It is used acutely with Haemophilus Influenzae infections, susceptible Acinetobacter infections, Polymicrobial soft tissue infections such as diabetic foot ulcers and postsurgical wound infections (Hviid et al., 2011). Ampicillin/Sulbactam is contraindicated for individuals having history of a Penicillin allergy (Zaza and Hopf, 2018). This study aimed to evaluate the effect of chronic administration of Unictam (broad spectrum antibiotic consisting of Ampicillin/Sulbactum) on the development \& mineralization of enamel of the mandibular first molars of Albino rats.

\section{MATERIALS AND METHODS}

This study was conducted after receiving an ethical clearance from the Research Ethics Committee of Ain Shams University, Faculty of Dentistry; that the study follows the guideline of research ethical committee code of practice for animal care and housing. Eight female Albino rats (250g weight) were housed in the Animal House Unit, Faculty of Medicine, Ain Shams University. They were kept there for one week for adaptation and observation. The females in estrus were mated overnight with males (two females per male). A vaginal smear was used to insure presence of sperms indicating the first sign of pregnancy.

The pregnant rats were divided into two groups $(n=4)$ : untreated (I) \& treated (II) groups which was injected intraperitoneally with $200 \mathrm{mg} / \mathrm{kg}$ body weight (Germiniani et al., 1992) Unictam (Ampicillin/Sulbactum from Almahan Altabya Pharmaceutical Company, Cairo.) from day 13 of pregnancy till delivery. Unictam was further 
intraperitoneally injected to their offsprings for additional 11 days. The treated offsprings from treated mothers were divided into two subgroups (IIA\&IIB) ( $\mathrm{n}=7$ each) which were sacrificed at 12 \& 28 day after birth respectively. The corresponding untreated subgroups (IA \&IB) ( $\mathrm{n}=7$ each) were sacrificed at same dates. The mandibular first molars of the subgroups were investigated by:

- Histopathological stain: The head of seven pups of subgroup IA \&IIA was fixed in $10 \%$ formalin for 24 hours, processed to be embedded in paraffin, sectioned in sagittal section to four microns and stained by H\&E to examine the longitudinal section in the tooth germ of mandibular first molars.

- $Q C T$ : Seven offsprings from subgroups IB\&IIB were sacrificed and fixed in formalin for $24 \mathrm{~h}$ then washed and stored in phosphate buffered saline. The samples were put into tubes to prevent their movement then carried on tube holder. Quantitative computerize tomography (QCT) was performed in Misr Radiology Center in Cairo to measure enamel mineral density. After the QCT analysis, heads of offspring were divided into two halves where the right mandibular halves were processed to be examined by ground section under light microscope (LM) and the left halves were processed to be examined by scanning electron microscope (SEM).

- Ground section: The right mandibular halves (carrying fully mineralized teeth) of subgroups IB\& IIB were processed and thinned in mesiodistally direction by a diamond disc into one $\mathrm{mm}$ thickness to examine the longitudinal section in the enamel of mandibular molar under L.M.

- Scanning Electron microscope: left halves of the mandibles of both subgroups IB\&IIB were cleaned by rubber cup and prepared to examine enamel of middle third of buccal surface of mandibular first molars by Quanta FEG 250 SEM.

\section{- Morphometric \& Statistical Analysis:}

a. Morphometric measurement of the enamel matrix thickness in H\&E sections for IA\&IIA subgroups was performed. The measurements were taken in four different positions: cusp tip, two middle positions \& cervical position.

b. Morphometric measurement of enamel thickness in ground sections of IB\&IIB subgroups was performed. The measurements were taken in four different positions: cusp tip, two middle positions \& cervical position.

c. For QCT analysis, the maximum and minimum densities of enamel of IB\&IIB subgroups were measured in the middle third of buccal surfaces of first molars of pups.

Mean, standard deviation and $\mathrm{p}$ value of the above data were calculated using "excel add in realstat v.5.3". Difference between two subgroups were analyzed by independent samples t-test where $\mathrm{p} \leq 0.05$ was considered statistical significant.

\section{RESULTS}

\section{Light microscopic results}

- H\&E stained sections of mandibular first molars at 12 days of subgroup IA presented normal architecture of tooth germ (fig. 1 A). Subgroup IIA showed an apparent reduction in enamel matrix thickness (fig.1B).

- Light microscopic examination of longitudinal ground section of mandibular first molars at 28 days of subgroup IB \& IIB (fig. 2A\&B) demonstrated the enamel and dentin separated by dentino-enamel junction (DEJ). Subgroup IIB (fig. 2B) showed an apparent thin layer of enamel. 


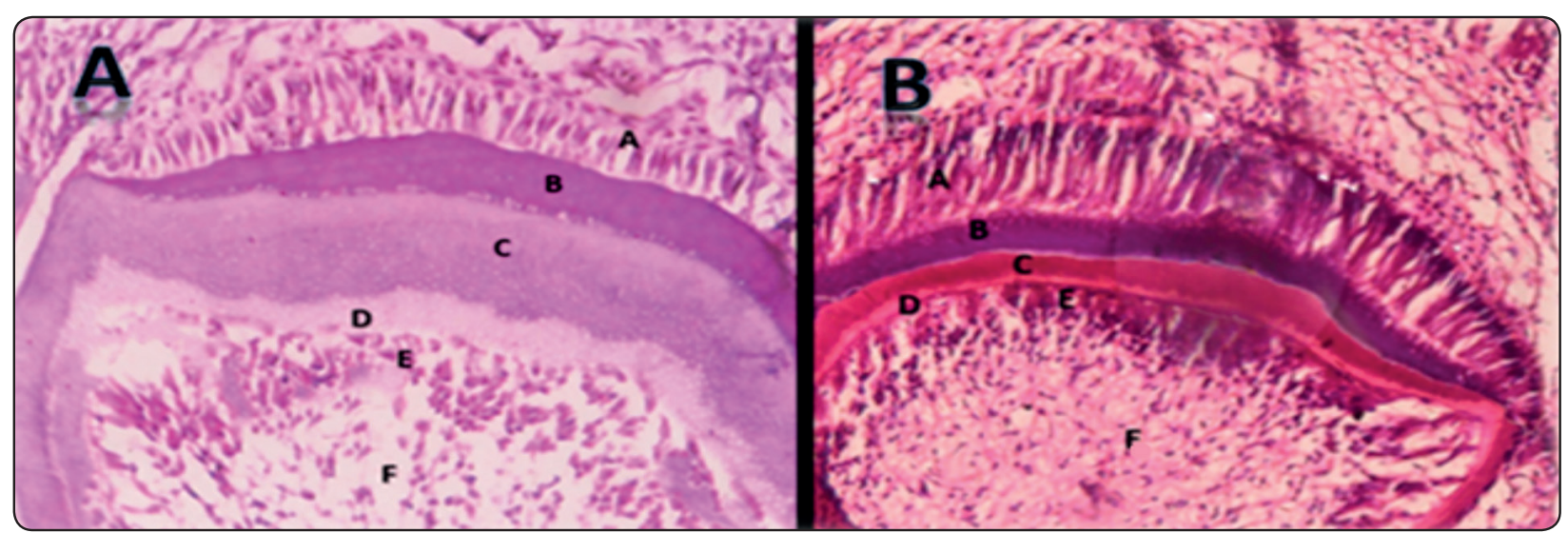

Fig. (1) A\&B: Photomicrographs of mandibular first molar tooth germ at 12 days of subgroups IA\&IIA respectively where: (A) tall columnar ameloblasts with polarized nucleus, (B) enamel matrix, (C) layer of dentin, (D) predentin, (E) odontoblasts. B: Note the apparent reduction in enamel matrix thickness of treated subgroup (B) (H\&E org. mag. x 400)

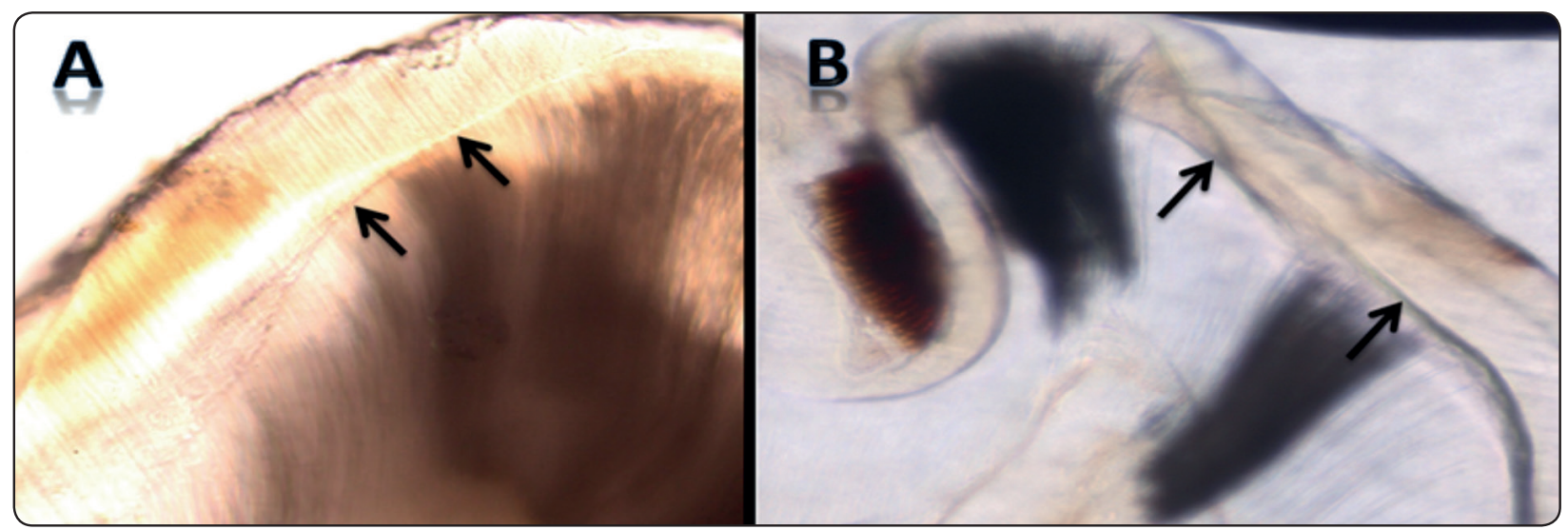

Fig. (2) A\&B: Photomicrographs of longitudinal ground section of mandibular first molar at 28 days of subgroups IB and IIB respectively showing enamel and dentin separated by DEJ (arrow). B: note the apparent reduction in enamel thickness (org. mag. $\mathrm{x}$ 400).

Scanning Electron Microscopic results: examination of subgroup IIB showed an irregular enamel surface with pores \& fissures (fig. $3 \mathrm{C} \& \mathrm{D}$ ) compared to smooth surface of control subgroup IB. (fig. $3 \mathrm{~A} \& \mathrm{~B}$ )

\section{Morphometric and statistical analysis:}

- Enamel matrix thickness in H\&E sections: The morphometric analysis for enamel matrix in H\&E stained section of both subgroups IA \& IIA revealed a statistically insignificant reduction of enamel matrix thickness of the molars in subgroup IIA when compared with subgroup IA. (table1,Fig.4)

- Enamel thickness in ground sections: The morphometric analysis of enamel thickness in the ground sections of both subgroups IB \& IIB revealed a reduction in the enamel thickness of the molars in subgroup IIB compared with the subgroup IB. However, the difference was statistically insignificant (table 2, Fig.5) 

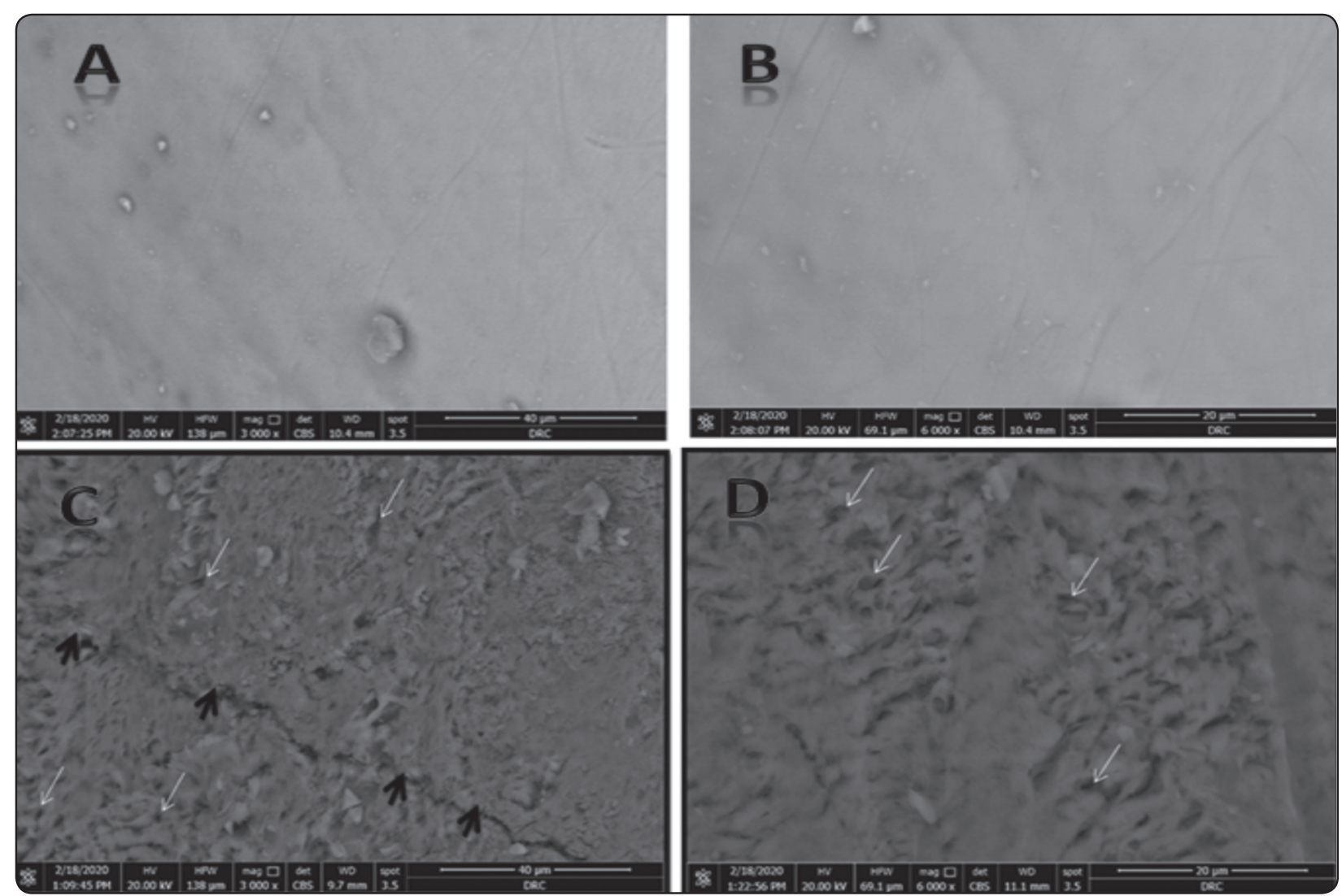

Fig. (3) A,B,C\&D: Scanning electron micrographs of buccal surface of rat mandibular first molar at 28 days of subgroups IB (A\&B) and subgroup IIB (C\&D). A\&B: smooth surface of enamel with little scratches. C\&D: rough surface with pores (white arrows) \& fissures (black arrows). (A\&C x3000 and B\&D x 6000)

TABLE (1) Mean, standard deviation \& p-value of thickness of enamel matrix of rat mandibular first molar for subgroups IA \& IIA.

\begin{tabular}{|l|c|c|l|}
\hline & Mean & \multicolumn{1}{|c|}{ SD } & \multicolumn{1}{c|}{ P value } \\
\hline subgroup IA & 241.7857143 & 40.6646418 & \\
\hline subgroup IIA & 238.1428571 & 32.5780655 & ns \\
\hline
\end{tabular}

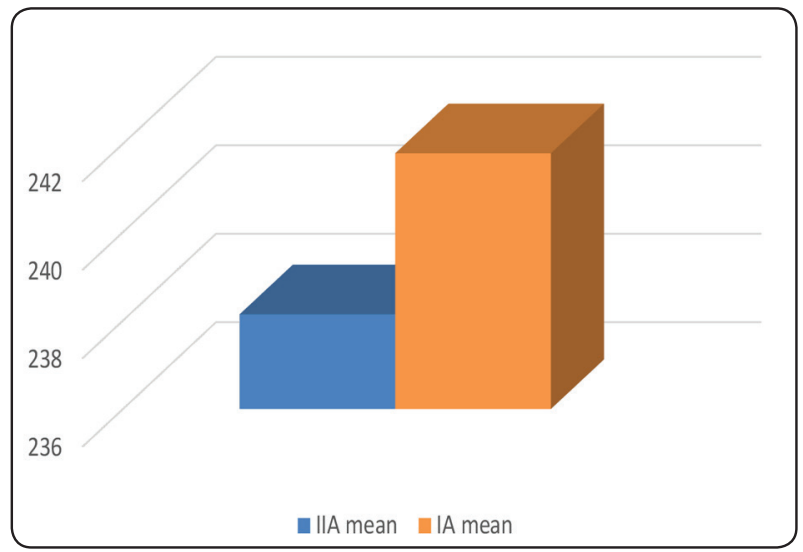

ns :non significant

Fig. (4) Bar char illustrating the mean of the thickness of enamel matrix of the first molar of Abino rat for subgroups IA \& IIA. 
TABLE (2) Mean, standard deviation \& p-value of thickness of fully mineralized enamel of rat mandibular first molar for subgroups IB \& IIB.

\begin{tabular}{|c|c|c|c|}
\hline & Mean & SD & P value \\
\hline subgroup IB & 242.9286 & 40.81506 & $\begin{array}{c}0.728778 \\
\text { ns }\end{array}$ \\
\cline { 1 - 3 } Subgroup IIB & 236.2143 & 28.96026 & \\
\hline
\end{tabular}

ns :non significant

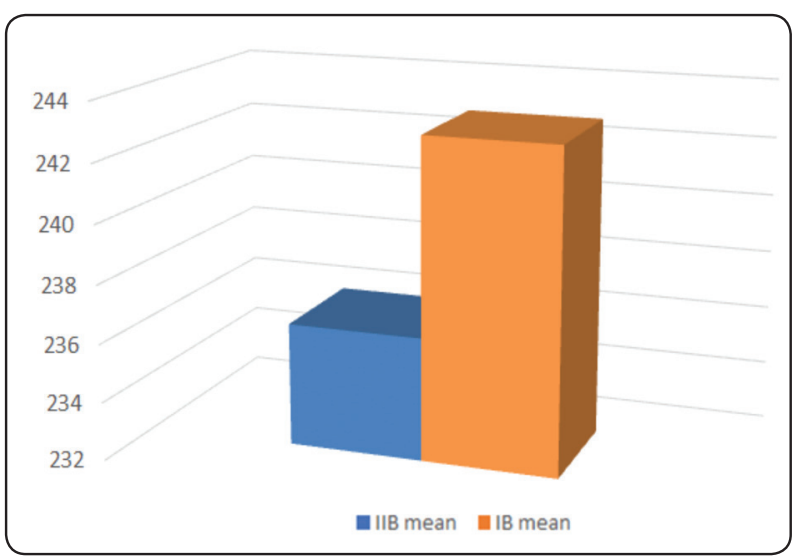

Fig. (5) Bar Chart illustrating the mean of the thickness of fully mineralized enamel of the first molar of Abino rat for subgroups IB \& IIB.

\section{- Quantitative Computerized Tomography}

(QCT) results revealed a statistically significant reduction in the density of enamel in subgroup IIB compared with subgroup IB (table 3, Fig.6).

TABLE (3) Mean, standard deviation \& p-value of density values of fully mineralized enamel of rat mandibular first molar for subgroups IB \& IIB.

\begin{tabular}{|l|c|c|c|}
\hline & Mean & SD & P value \\
\hline Subgroup IB & 818.1429 & 24.05326431 & \multirow{2}{*}{$0.002275669^{*}$} \\
\hline Subgroup IIB & 768.0714 & 24.50242942 & 0.006 \\
\hline
\end{tabular}

*:significant

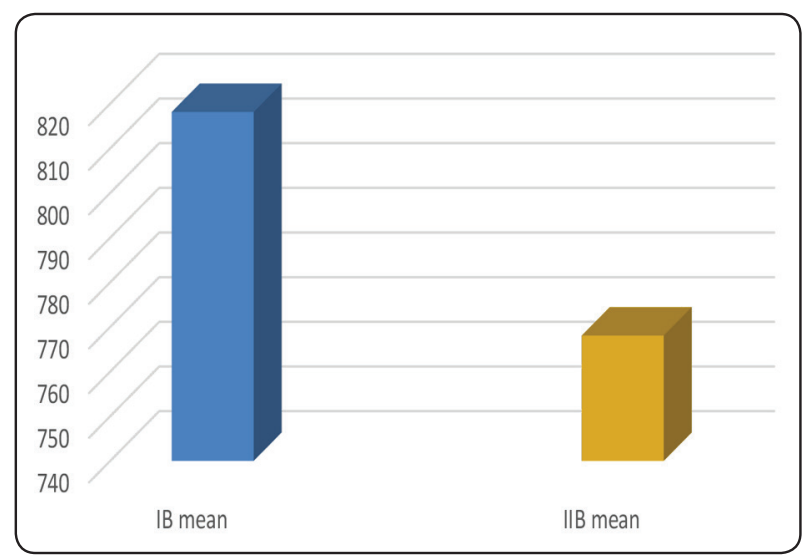

Fig. (6) Bar Chart illustrating the mean of the enamel density of the first molar of Abino rat for subgroups IB \& IIB.

\section{DISCUSSION}

The prevalence of molar-incisor hypo mineralization was reported to vary from $2.4 \%$ to $40.2 \%$ in different countries (Koruyucu et al., 2018).

Unictam (Ampicillin/Sulbactam), considered as a safe antibiotic was the drug of choice in this study instead of Ampicillin alone since it was reported to be more effective and more prescribed during pregnancy (Fallon et al., 2005).

Albino rats were chosen in this study since their teeth have the same developmental stages as humans which facilitate the investigation of antibiotic effects on tooth enamel development at any time during the animal's life (Bronckers et al., 2009).

In the present work, molars were selected rather than incisors as rats use their incisors in gnawing and cutting so they are subjected to more friction, in addition to the fact that they undergo growth throughout life (Martin et al., 2016).

Intraperitoneal injection was selected in this study as route of drug administration instead of oral route to avoid pre-systemic degradation of Ampicillin in the intestine of small rodents due to lack of hepatic first pass metabolism for Ampicillin (Ali et al., 2012). 
In the present work, Unictam was given from the $13^{\text {Th }}$ day of rats' pregnancy which corresponds to initiation period of molar (Simmer et al., 2010). The subgroups IA\&IIA were sacrificed on $12^{\text {th }}$ day after birth to observe first molar enamel matrix before its final mineralization (DenBesten et al., 2002). Subgroups IB\&IIB were sacrificed on $28^{\text {th }}$ day after birth where a full set of teeth was fully erupted (Rios et al., 2005; Catón and Tucker, 2009) which allows the investigation of molars'fully mineralized enamel with ground sections, SEM \& QCT.

In the present work, The H\&E stained sections revealed an apparent decrease in enamel matrix thickness in subgroup IIA compared to subgroup IA. However, the statistical analysis revealed that this decrease was insignificant which is not in agreement with the study Sahlberg et al. (2013) revealing a significant decrease in enamel matrix thickness upon administration of Amoxicillin (a member of the Beta lactam antibiotic family) to mice. The controversy in results could be attributed to the small sample size used in the present study compared to Sahlberg's study.

On the $28^{\text {th }}$ postnatal day, SEM revealed pores and fissures on the surface of subgroup IIB compared to almost smooth regular surface in subgroup IB. These findings moved along with those Sahlberg et al. (2013) where defects in enamel surface were detected in rats treated with Amoxicillin. The investigators suggested that Amoxicillin affected ameloblasts during the development of enamel producing rough, foamy appearance in the affected areas of enamel as well as porosities of the enamel surface.

In the present study, QCT results of mandibular first molar of subgroup IIB revealed a statistically significant reduction in enamel density compared with subgroup IB indicating hypomineralization as reported by Kuscu et al., in 2013. The investigator detected significant decrease in molar enamel mineral density in Amoxicillin-treated piglets compared to control group using X-ray micro-tomography, they considered the decrease in enamel density as a sign of hypomineralization.

The results of QCT of the present study are also in parallel with those of Jälevik et al. (2001) through Energy Dispersive Analysis X-Ray (EDAX) where Amoxicillin \& Clavulanic acid (a member of the Beta lactam antibiotic family) treated groups tended to show a lower content of $\mathrm{Ca}$ and $\mathrm{P}$ in enamel, indicating its hypomineralization. (Wuollet et al., 2016) also proved the association of antibiotics (especially amoxicillin) intake with hypomineralization of the permanent molars and incisors of 287 Finnish children through their medical records.

On the other hand, Alaluusua et al. (2010) concluded that the molar-incisor hypomineralization prevalence didn't differ between children who received antibiotics during their first three years and those who didn't. Their clinical retrospective study was based on the data collected from questionnaires, interviews \& medical records. The controversy in our results with Alaluusa 's study could be referred to lack of specific hypomineralization assessment and the dependence on collected data.

Kumazawa et al. (2012) suggested that a single dose of $3 \mathrm{~g} / \mathrm{kg}$ Amoxicillin affected normal tooth dentin mineralization, but not enamel mineralization in Wistar rat incisor odontogenesis. This difference in results with the present work may be attributed to Amoxicillin single heavy dose usage compared to long duration of lower dose of Unictam administration in our study.

Based on the results of the present study, it could be concluded that chronic administration of Unictam in Albino rats led to disturbances in the development and mineralization of enamel of mandibular first molar of Abino rats. 


\section{REFERENCE}

- $\quad$ Ali B., Amin S.,Ahmad J., Ali A., Ali M., \& Mir S. R. (2012): Bioavailability enhancement studies of amoxicillin with $\mathrm{Ni}$ gella. Indian Journal of Medical Research; 135(4):555-559.

- $\quad$ Allazzam S. M., Alaki S. M., \& El Meligy O. A. S. (2014): Molar incisor hypomineralization, prevalence, and etiology. International Journal of Dentistry, 2014: 1-8.

- Catón J., \& Tucker A. S. (2009): Current knowledge of tooth development: patterning and mineralization of the murine dentition. Journal of Anatomy; 214(4): 502-515.

- DenBesten P. K., Yan, Y., Featherstone J. D. B., Hilton J. F., Smith C. E., \& Li W. (2002): Effects of fluoride on rat dental enamel matrix proteinases. Archives of Oral Biology; 47(11): 763-770.

- Fallon L., Jadwat Y., Bouckaert M., Buskin A. \& Raubenheimer E.J. (2005): Enamel dysplasia with odontogenic fibroma-like hamartomas; Review of the literature and report of a case. Oral Surg, Oral Med Oral Pathol Oral Radiol and Endo; 101(5):620-624

- $\quad$ Elina Nyström M., Meri Ranta, H., Sakari Peltola, J., \& Matti Kataja, J. (2007): Timing of developmental stages in permanent mandibular teeth of Finns from birth to age 25 . Acta Odontologica Scandinavica;65(1):36-43.

- Germiniani, R., Casiraghi, S., Pace, M., Caspani, P., \& Trabucchi,E. (1992): Sulbactam-Ampicillina in chirurgia. la nostra esperienza. Minerva Chirurgica, 47(20), 1627-1629.

- Hviid A., Svanström H. and Frisch M. (2011): Antibiotic use and inflammatory bowel diseases in childhood. Gut;60(1):49-54

- Jälevik B., Norén J.G., Klingberg G. \& Barregård L. (2001): Etiologic factors influencing the prevalence of demarcated opacities in permanent first molars in a group of Swedish children. Eur. J. Oral Sci;109(4):230-234.

- $\quad$ Koruyucu M., Özel S., \& Tuna E. B. (2018): Prevalence and etiology of molar-incisor hypomineralization $(\mathrm{MIH})$ in the city of Istanbul. Journal of Dental Sciences: 13(4); 318-328.
- Kumazawa Kaido, Takashi Sawada, Takaaki Yanagisawa, and Seikou Shintani. (2012): Effect of single-dose Amoxicillin on Rat Incisor Odontogenesis: A Morphological Study. Clinical Oral Investigations; 16(3):835-842.

- Kuscu O. O., Sandalli N., Dikmen S., Ersoy O., Tatar I., Turkmen İ., \& Çaglar E. (2013): Association of amoxicillin use and molar incisor hypomineralization in piglets: Visual and mineral density evaluation. Archives of Oral Biology; 58(10): 1422-1433.

- Rao M. H., Aluru S. C., Jayam C., Bandlapalli A., \& Patel N. (2016): Molar incisor hypomineralization. Journal of Contemporary Dental Practice;17(7): 609-613.

- Sahlberg C., Pavlic A., Ess A., Lukinmaa P.-L., Salmela E., \& Alaluusua S. (2013): Combined effect of amoxicillin and sodium fluoride on the structure of developing mouse enamel in vitro. Archives of Oral Biology; 58(9): $1155-1164$.

- Sengupta P. (2013): The Laboratory Rat: Relating Its Age with human's. International Journal of Preventive Medicine; 4(6): 624-630.

- $\quad$ Simmer J. P., Papagerakis P., Smith C. E., Fisher D. C., Rountrey A. N., Zheng L., \& Hu J. C. C. (2010): Regulation of dental enamel shape and hardness. In Journal of Dental Research; 89(10): 1024-1038.

- Thesleff I., Keranen S., \& Jernvall J. (2001): Enamel knots as signaling centers linking tooth morphogenesis and odontoblast differentiation. Advances in Dental Research; 15(1): 14-18.

- Wuollet E., Laisi S., Salmela E., Ess A. \& Alaluusua S. (2016): Molar-incisor hypomineralization and the association with childhood illnesses and antibiotics in a group of Finnish children. Acta Odontol Scand; 74(5): 416-422.

- Zaza Khaled J. and Harriet W. Hopf. (2018): Infection, Antimicrobial Drugs, and Anesthesia. Pharmacology and Physiology for Anesthesia: Foundations and Clinical Application;2018: 769-780. 